\title{
Phase Messaging Method for Time-of-flight Cameras
}

\author{
Wenjia Yuan ${ }^{1}$, Richard E. Howard ${ }^{1}$, Kristin J. Dana ${ }^{1}$, Ramesh Raskar ${ }^{2}$ \\ Ashwin Ashok ${ }^{1}$, Marco Gruteser ${ }^{1}$, Narayan Mandayam ${ }^{1}$ \\ Rutgers University, WINLAB ${ }^{1} \quad$ MIT Media Lab $^{2}$
}

\begin{abstract}
Ubiquitous light emitting devices and low-cost commercial digital cameras facilitate optical wireless communication system such as visual MIMO where handheld cameras communicate with electronic displays. While intensity-based optical communications are more prevalent in camera-display messaging, we present a novel method that uses modulated light phase for messaging and timeof-fight (ToF) cameras for receivers. With intensity-based methods, light signals can be degraded by reflections and ambient illumination. By comparison, communication using ToF cameras is more robust against challenging lighting conditions. Additionally, the concept of phase messaging can be combined with intensity messaging for a significant data rate advantage. In this work, we design and construct a phase messaging array (PMA), which is the first of its kind, to communicate to a ToF depth camera by manipulating the phase of the depth camera's infrared light signal. The array enables message variation spatially using a plane of infrared light emitting diodes and temporally by varying the induced phase shift. In this manner, the phase messaging array acts as the transmitter by electronically controlling the light signal phase. The ToF camera acts as the receiver by observing and recording a time-varying depth. We show a complete implementation of a $3 \times 3$ prototype array with custom hardware and demonstrating average bit accuracy as high as $97.8 \%$. The prototype data rate with this approach is 1 Kbps that can be extended to approximately 10 Mbps.
\end{abstract}

\section{Introduction}

As cameras become ubiquitious, communication between displays and cameras has become an active research area. Methods of Visible-Light Communication (VLC) have been developed where standard cameras detect patterns intentionally placed within images. These patterns can be fixed as in QR-codes [14], dynamic as in Visual MIMO, [4,27] or LED-to-LED applications [22], and coded using OFDM as in Pixnet $[17,21]$. The main theme in these

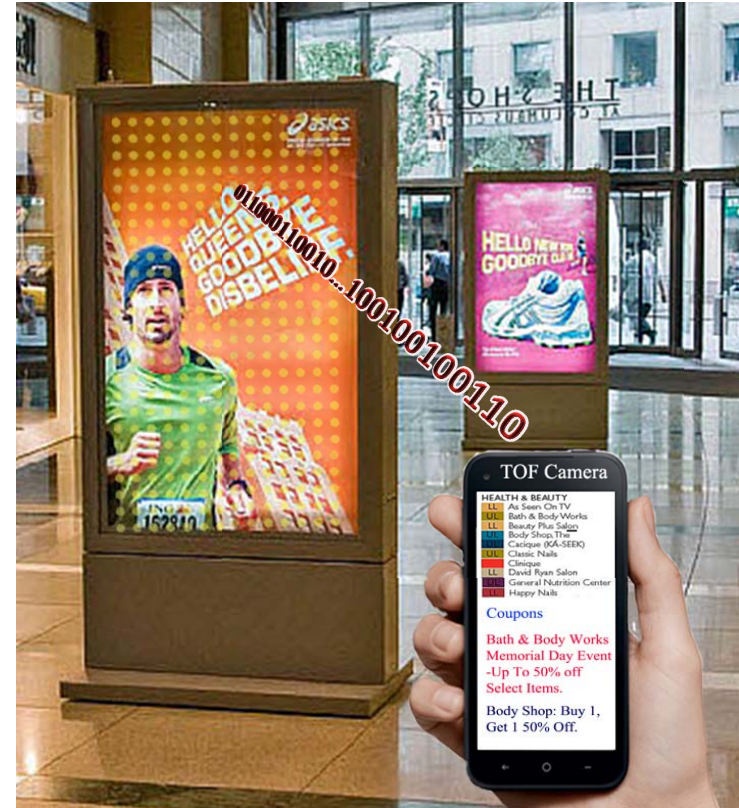

Figure 1. Concept illustration of "smart signage" for communicating to cameras in the environment with infrared LEDs that send invisible messages for communication with time-of-flight depth cameras. These cameras are increasingly more commonplace and can be expected in mobile robotics and devices. Large LEDs are shown for illustration clarity, but small infrared LEDs would be unnoticeable in a real application.

methods is communication by modulating light intensity from an observed image. Recently, high quality depth cameras using time-of-flight (ToF) have become readily available at low-cost, creating an entirely new opportunity for communications. Creating a depth-pattern is a more challenging optical task when compared to intensity patterns. However, since depth is measured with ToF cameras using the modulated phase of reflected light, manipulating this light signal effectively changes the measured depth, resulting in a phase-based message.

The applications for depth cameras are numerous since most intelligent sensing applications can benefit from scene geometry information. We develop a novel method for com- 


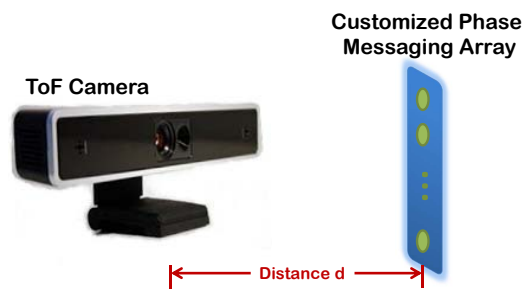

(a)

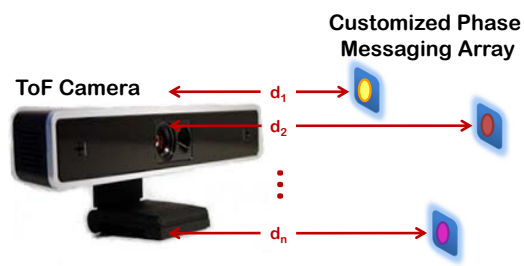

(b)

Figure 2. Phase Messaging Array (PMA) method. (a) A customized PMA with $\mathbf{n}$ LEDs is physically at distance $\mathbf{d}$ away from a ToF camera. (b) By modulating phases for LEDs on PMA, LEDs "seem" at individual distances away in depth images from the ToF camera. Messages are represented by these measured distances.

municating with depth cameras using the phase of infrared (IR) light modulation signal. The transmitter arrays can be used as beacons, tags or communication channels that interact with RGB-D cameras for real world applications. Specific applications include: "smart signage" with invisible light messaging to cameras in the environment (see Figure 1); vehicle id tags transferring information to road cameras or cameras on other vehicles; robots that gather task information like access keys stored in fixed beacons; product tags with information (shelf life, expiration date and price) that are read during scene depth-imaging so that the product can also be localized.

While visible light communication has become a popular research area, ambient illumination and reflections in real world environments can degrade the signal quality. Messaging with light phase instead of intensity has distinct advantages. First, time-of-flight cameras are designed to measure the phase of the modulated source light signal (typically $15-30 \mathrm{MHz}$ ) by correlating the source and the reflected signal. This design means that depth cameras are tuned to their source light signal and are relatively unaffected by environment lighting. Therefore, reflections and ambient lighting do not degrade the quality of the signal. This degradation is a major problem for operations of intensity-based systems. Additionally, the time-of-flight camera measures time delays on the order of nanoseconds, and this short time interval makes the method particularly useful for mobile scenarios where the camera and messenger are in motion (e.g. handheld camera, automotive applications). The time interval of the light path is orders of magnitude smaller than that of the system motion, so that motion blur is not an issue. On the contrary, intensity-based messaging is vulnerable to degradation by motion blur. Furthermore, the use of IR light enables messaging without visibility to human observers.

In this work, we design and construct a phase messaging array (PMA), which is the first of its kind, to communicate to a depth camera by manipulating the phase of the depth camera's own light signal (see Figure 2). We use the term light signal to refer to the modulation of light as described in Section 3.1, and the phase of this modulated signal is modified. The light signal is replicated and the phase is shifted by our custom circuitry. Replicating the camera's own light signal is a key design component of the system. The timeof-flight camera is generally unresponsive to light signals that are not exactly matched in frequency to their source signal. Sensing and replicating the original camera signal with a message encoded in the phase shift ensures independence of the transmitter (PMA) and receiver (camera) in the communications channel. A spatial distribution of IR light emitting diodes allows messaging units to be packed in the $\mathrm{x}-\mathrm{y}$ plane for an increased data rate. The array driving circuitry measures the depth camera's source signal and effectively delays that signal according to the desired message. In this manner, the depth camera observes a time-varying depth that is electronically controllable. We show a prototype implementation of a $3 \times 3$ array with 4 phase symbols and messaging accuracies around $97.8 \%$. The data rate of this $3 \times 3$ array approaches $1 \mathrm{Kbps}$ which can be readily extended to a data rate more than $10 \mathrm{Mbps}$ (see our discussion in Section 4.2).

\section{Related Work}

Visible-Light Communications (VLC) is a general term for methods that use visible light instead of radio frequencies for communication. A theme in VLC in recent years has been to extend existing infrastructure to create new communication channels. Li-Fi [9] uses light-bulbs that not only illuminate the room, but can also transmit temporally dynamic data by modulating light intensity. The data rate of $\mathrm{Li}-\mathrm{Fi}$ with a single color LED reaches $1.67 \mathrm{Gbps}$; however, specialized equipment is needed for both the receiver and transmitter. Increasing the field-of-view in visible light communications has been addressed in [25] with the use of hemispherical lenses at the receivers. However, the use of ordinary cameras provides the benefit of an inherently wide field-of-view. Visual MIMO [3,4] is a method that uses any light-emitting spatial array such as an LCD display as the transmitter and uses ordinary cameras as the receivers. This method is a type of Camera-Display Communication (CDC) since the framework enables cameras and electronic displays to communicate. Other CDC methods include QR-codes [1,14], Bokode [16] and Pixnet [21]. QR-codes are comprised of black-white block patterns, and are quite common in recent years applied to existing ads 
or signage. Unlike Visual MIMO, the QR-code is passive so the information is static and the pattern is visible to the human observer. Similar to Visual MIMO, QR-codes are designed for use with general purpose cameras, allowing broad real-world applicaton. Bokode and Pixnet also display message codes with light-emitting devices as transmitters. The difference is that Bokode exploits the bokoh effect of ordinary camera lenses for long-distance static tag reading, while Pixnet generalizes the popular OFDM transmission algorithms $[2,15]$ to address the unique characteristics of the LCD-camera link. Visual MIMO [27-29] proposes a photometric modeling for hidden imagery and can realize a dual use the electronic display: to display an image for human observation while simultaneously transmitting hidden bits for decoding by a camera. The hierarchy of this work in optical communications in real-world environments progresses from barcodes [18] that are one-dimensional and static, to QR-codes that are two-dimensional and static, to the various CDC methods that use controllable intensity patterns and are two dimensional and time-varying. Our method adds to this framework with phase messaging that uses controllable phase patterns for 2D time-varying messaging. We continue the theme of using ordinary cameras for messaging since low-cost unmodified ToF cameras are used for the receiver.

Ordinarily, ToF cameras are used to directly estimate 3D scene structure complementing traditional multiple-view reconstruction methods $[10,11]$. Applications using ToF cameras include 3D scene reconstruction [6,23], reflectance capture using ultrafast imaging [20], frequency analysis to address multipath interference $[5,13,26]$ and human pose detection/tracking $[7,8]$. These depth cameras are increasingly common in various application domains such as intelligent vehicles, robotic environment mapping and surveillance. Our novel framework deploys ToF depth cameras as receivers for optical communications. Compared with intensity-based messaging methods, this phase-based messaging is more robust against background illumination as shown in our experimental results. Also, high measurement precision in phases (Softkinetic ToF cameras have 32768 phase levels) can create a larger coding space for an increased data rate.

\section{Methods}

\subsection{Introduction to Time-of-flight Camera}

We summarize the basic operation of ToF cameras as presented in $[12,19]$. While most intensity-based digital camera are passive, ToF cameras rely on integrated light sources. During imaging, photons emitted from this light source travel to objects in the scene. After surface reflection, the returning light is sensed by the ToF camera. The light travels a distance equal to twice the object depth relative to the camera. There are two main types of ToF cam-

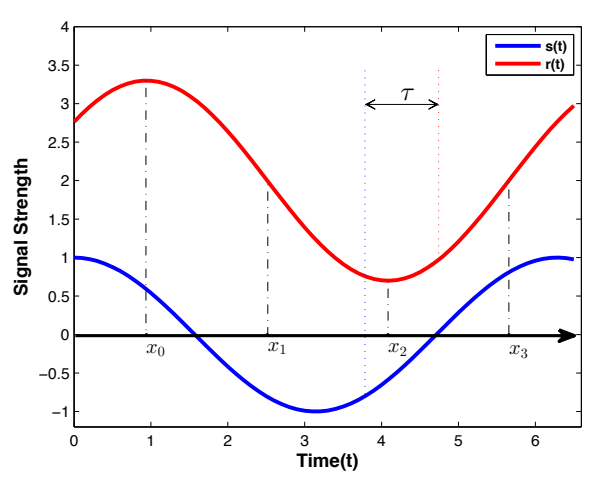

Figure 3. Four bucket method. Get four equidistant samples of the received signal $r(t)$ within one cycle. Their cross-correlations with the sent signal $s(t)$ are $c\left(x_{0}\right), c\left(x_{1}\right), c\left(x_{2}\right), c\left(x_{3}\right)$. They can be used to solve the depth, which is proportional to the delay $\tau$.

eras: (1) pulse-modulation (PM) (2) continuous-wave (CW) For this work, we use a continuous wave ToF camera using IR LEDs, since they are readily available at low cost. ${ }^{1}$

Denote the modulation frequency as $f_{m}$. The sent signal $s(t)$ and the received signal $r(t)$ with delay $\tau$ are given by

$$
\begin{aligned}
& s(t)=a \cos \left(2 \pi f_{m} t\right), \\
& r(t)=A \cos \left(2 \pi f_{m}(t-\tau)\right)+B
\end{aligned}
$$

where $A$ and $B$ are the unknown scale and offset of the reflected signal and these parameters are estimated at the camera. For $s(t)$ and $r(t)$, the cross-correlation is

$$
\begin{aligned}
c(x) & =\lim _{T \rightarrow \infty} \int_{-\frac{T}{2}}^{\frac{T}{2}} r(t) s(t+x) d t \\
& =\frac{a A}{2} \cos \left(2 \pi f_{m} x+2 \pi f_{m} \tau\right)+B .
\end{aligned}
$$

There are three unknown variables in Eqn 3: amplitude $A$, offset $B$ and delay $\tau$. These variables can be solved by "the four-bucket method" [12,19]. The basic principle is to obtain 4 equidistant samples at locations $\left(x_{0}, x_{1}, x_{2}, x_{3}\right)$ within a modulation cycle as in Figure 3. The crosscorrelations of these four outputs with the source signal $s(t)$ is denoted by $c\left(x_{0}\right), c\left(x_{1}\right), c\left(x_{2}\right), c\left(x_{3}\right)$. The three unknown variables can be solved based on these correlations in closed form. The amplitude $A$ can be solved as

$$
A=\frac{\sqrt{\left[c\left(x_{3}\right)-c\left(x_{1}\right)\right]^{2}+\left[c\left(x_{0}\right)-c\left(x_{2}\right)\right]^{2}}}{2} .
$$

The offset $B$ is mainly attributed to background illumination and can be solved as

$$
B=\frac{c\left(x_{0}\right)+c\left(x_{1}\right)+c\left(x_{2}\right)+c\left(x_{3}\right)}{4} .
$$

\footnotetext{
${ }^{1}$ Creative Interactive Gesture Camera Developer Kit from Intel costs \$149. DepthSence311 from SoftKinetic costs \$299. (December 2013)
} 


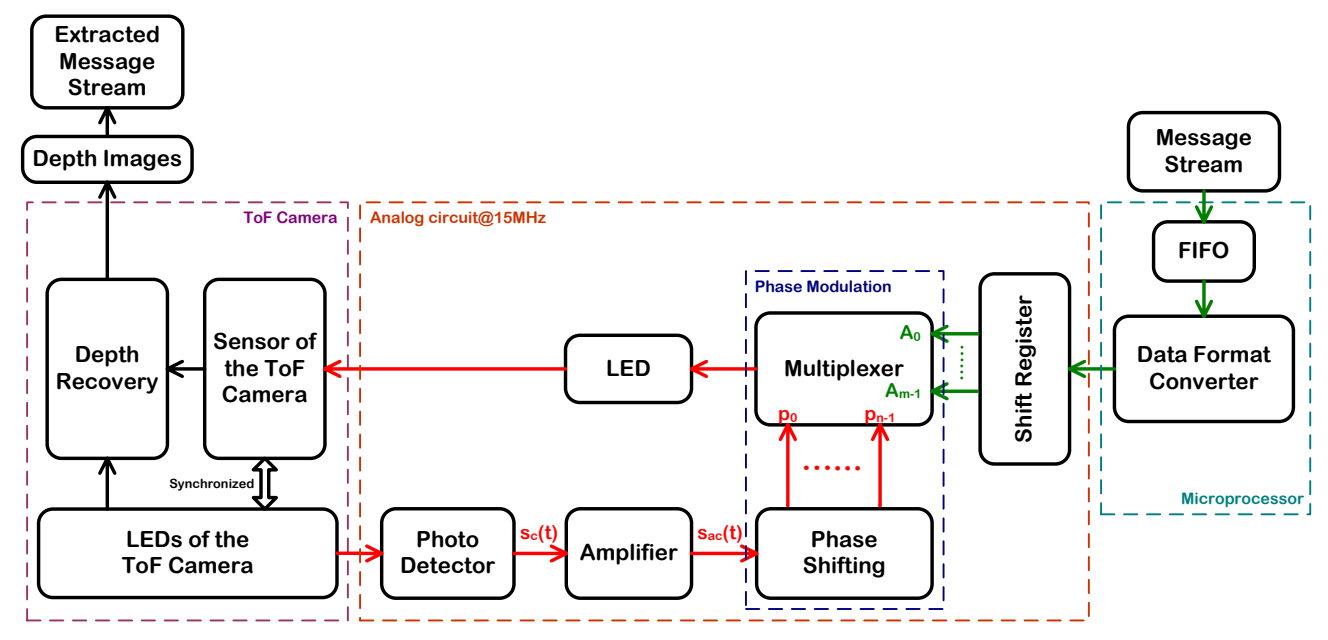

Figure 4. Basic Operating Principle of the Phase Messaging Array (PMA). For clear illustration of the method, only one transmitter LED is shown. Data flow is labeled with red arrows and control flow is in green. As a communications channel, the LED's on the PMA are the message transmitters and the ToF camera is the receiver. The PMA senses the signal from the ToF LED light source using a photo detector. This copy of the light signal is denoted $s_{c}(t)$. After amplification, $s_{a c}(t)$ is used to create multiple phase-delay versions $p_{0}, \cdots, p_{n-1}$. Delays range in $(0,2 \pi]$ and are achievable in our design. These phase delays are symbols sent to multiplexers as output candidates. The control flow is the bit version of the message stream. At the multiplexer, the combination $\left[A_{m-1}, \cdots, A_{0}\right]$ selects the corresponding phase-delayed candidate signals to sequentially drive the transmitter LED in the next cascade.

The delay $\tau$ is the time of flight for light sent from camera LED, reflected from object's surface and received by the ToF camera and can be solved as

$$
\tau=\arctan \left[\frac{c\left(x_{3}\right)-c\left(x_{1}\right)}{c\left(x_{0}\right)-c\left(x_{2}\right)}\right] .
$$

The value of $\tau$ is proportional to the distance $d$ between the ToF camera and the captured object as $\tau=\frac{2 d}{c}$, where $c$ is light speed. Then the delayed phase between $s(t)$ and $r(t)$ can be denoted as $\phi=2 \pi f_{m} \tau=2 \pi f_{m} \frac{2 d}{c}$. Since $\phi$ ranges in $(0,2 \pi]$, the measured distance $d$ reaches its maximum $d_{\max }$ when $\phi=2 \pi$. For a ToF camera working at $f_{m}=$ $15 \mathrm{MHz}, d_{\max }=10 \mathrm{~m}$.

\subsection{Phase Messaging Array Method}

Controllable Phase Delay The design of our proposed PMA method is illustrated in Figure 4. For simplicity, the figure shows the basic operating principle with a single LED on the phase messaging array. As a communications channel, the PMA is a message transmitter and the ToF camera is the message receiver. The goal is to create phase delays at the PMA circuit that mimic depth variations in order to message to the ToF depth camera. Delaying light directly would typically require variation of the refractive index of the medium, and controllable delays in this manner are not practical. However, as an electrical signal, we can readily introduce delays using an appropriately designed cascade of logic gates. This approach is a key innovation in the design. We sense the signal from the source LED of the ToF camera using a photodiode to convert the light signal into the electrical signal $s_{c}(t)$. This copied light signal is amplified to $s_{a c}(t)$ and delayed to multiple phase-delay versions $p_{0}, \cdots, p_{n-1}$. Delays range in $(0,2 \pi]$ and are controllable in our design. In this manner, we create a dictionary of phase symbols and that are sent to the multiplexers as output candidates. The signals $s_{c}(t), s_{a c}(t)$ and $p_{i}(t)$ comprise the data flow component of the $P M A$ circuit. The control flow is the bit version of the message stream. At the multiplexer, the combination $\left[A_{m-1}, \cdots, A_{0}\right]$ selects corresponding phase symbol in a sequential way to drive the transmitter LED in the next cascade ${ }^{2}$.

There is a simple intuitive method of achieving the phase delay that fails in practice: applying a signal generator or crystal to produce signals at the same modulation frequency $f_{m}$ of the ToF camera. For the "four bucket method", it is critical to make sure the sent signal $s(t)$ and the received signal $r(t)$ have the same modulation frequency $f_{m}$. Though labeled with the fixed frequency value $f_{m}$, a signal generator or crystal always produces outputs with some variance around $f_{m}$. This frequency error converts into a delayed phase error and makes the measured depth values random. We have shown this experimentally in Figure 5(a) with the following procedure: A signal generator produces a sinusoid waveform at $15 \mathrm{MHz}$. This source drives an IR LED facing to a receiver ToF camera working at the same modulation frequency $f_{m}=15 \mathrm{MHz}$. As expected, the

\footnotetext{
${ }^{2} A_{m-1}$ is the Most Significant Bit (MSB), while $A_{0}$ is the Least Significant Bit (LSB).
} 


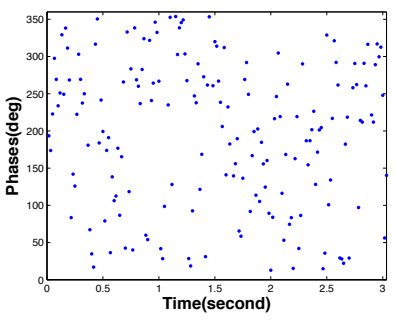

(a) Source: signal generator

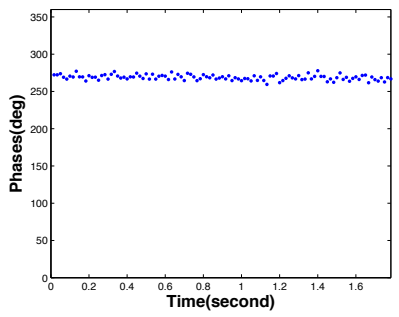

(b) Source: copied signal

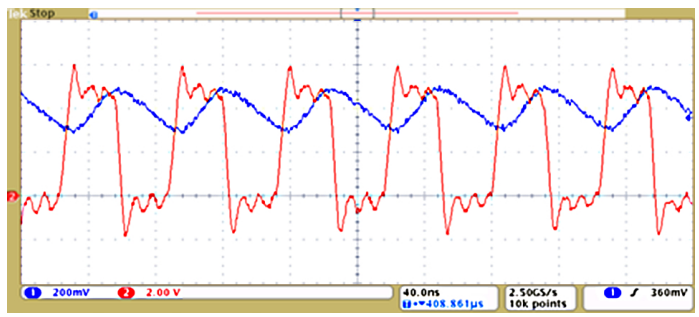

(c) Signal amplification

Figure 5. Comparison of two sources. (a) Measured phases of an IR LED driven by a $15 \mathrm{MHz}$ output from a signal generator are random, because of the frequency noise of the signal generator. (b) Measured phases of an IR LED by our proposed PMA method are stable. (c) Amplification of $s_{c}(t)$ in blue to $s_{a c}(t)$ in red.

measured phases of the LED from the depth image are random.

Our solution is to sense and copy the source signal from LED of the receiver ToF camera. An example is illustrated in Figure 5(c): the copied signal $s_{c}(t)$ in blue has an amplitude of $400 \mathrm{mV}$ with unit voltage $200 \mathrm{mV}$; the amplified signal $s_{a c}(t)$ in red has an amplitude of $5 \mathrm{~V}$ with unit voltage $2 \mathrm{~V}$. This amplified signal $s_{a c}(t)$ drives an LED facing the same ToF camera. Compared with Figure 5(a), the measured phases of the LED in Figure 5(b), are stable enough for messaging.

Phase Shifting For messaging, the mimicing signal of an LED from the receiver ToF camera is amplified into $s_{a c}(t)$. In our proposed scheme in Figure 6(a), there are four phasedelayed candidates: $p_{0}, p_{1}, p_{2}$ and $p_{3}$. Delays range in $(0,2 \pi]$ and are controlled by capacitors $C_{1}, C_{2}$ and $C_{3}$.

Each phase candidate is connected to the same channel of all multiplexers and each multiplexer drives one transmitter LED. Since each multiplexer works as a "switch", the configuration is a heavy load to output inverters $I n v_{0}$, $I n v_{a}, I n v_{b}$ and $I n v_{c}$, and may degrade the output signals severely. To prevent signal deterioration between cascades, the output signal $p_{i}$ for each cascade and the one sent to the next cascade are not the same. Additionally, inverter $I n v_{0}$ is used to increase driving ability of the input signal.

Figure 6(b) illustrates four phase images of an IR LED from the receiver ToF camera. Though the location of the

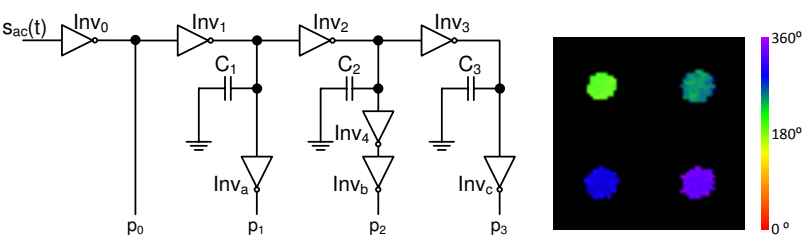

(a) Phase shifting scheme

(b) Four phase images

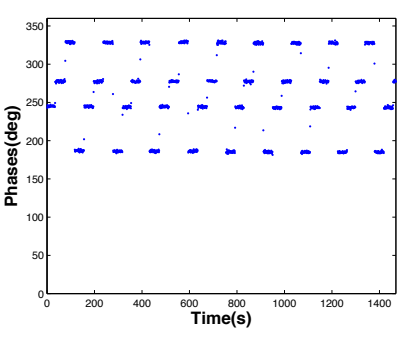

(c) Plot of phases

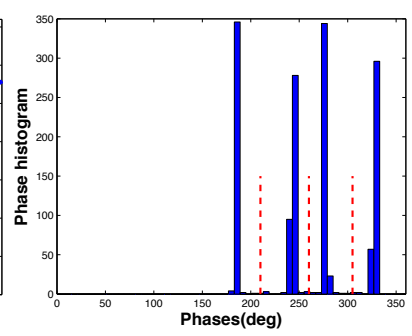

(d) Histograms of phases
Figure 6. Phase shifting. (a) In the phase shifting scheme, "deterioration prevention" and "phase delay control" are two basic considerations. (b) Four phase images of an LED displayed with delayed signals $p_{0}, p_{1}, p_{2}$ and $p_{3}$. Phase values are mapped to colors for visualization. (c) Plot of measured phases for an LED displayed with four delayed signals in turn. (d) Histogram of measured phases in (c). Red dotted lines are hard thresholds for phase symbol assignment.

LED is fixed, ToF camera measures it at four possible distances, corresponding to four phases $p_{0}, p_{1}, p_{2}$ and $p_{3}$. For visualization, these four candidate phases are mapped to individual colors. These four delayed signals are displayed sequentially on an IR LED controlled by a timer as in Figure 6(c). The histograms of all measured phases are shown in Figure 6(d). The histograms show the presence of outliers from the phase symbols due to transition states. We currently remove outliers by using an indicator LED to temporally identify transition times. Omitting transition times, the measured phase has a standard deviation $\sigma_{p}=1.2$ from an individual LED. The phases can be separated by hard thresholds indicated by the red dotted lines.

Messaging and Signal Selection Each of four delayed phases $p_{0}, p_{1}, p_{2}$ and $p_{3}$ (in red) are connected to the same channel of all multiplexers. Every multiplexer drives one transmitter LED facing towards the receiver ToF camera. The bit streams from the control flow (in green) determines which candidate to select.

One multiplexer case is shown in Figure 7(a). By the truth table in Figure 7(b) [24], signal $A_{1} A_{0}=10_{2}$ selects the signal with phase $p_{2}$. In the decoding scheme, messages are extracted by converting the measured phase value into the corresponding binary code using the truth table in Figure 7(b). 


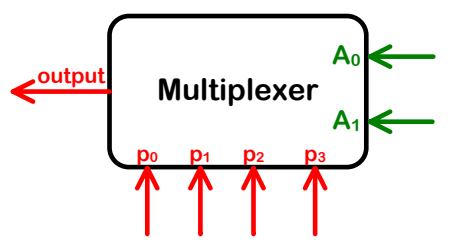

(a) Multiplexer

\begin{tabular}{cc|c}
$A_{1}$ & $A_{0}$ & output \\
\hline 0 & 0 & $p_{0}$ \\
0 & 1 & $p_{1}$ \\
1 & 0 & $p_{2}$ \\
1 & 1 & $p_{3}$
\end{tabular}

(b) Truthtable
Figure 7. Signal selection for messaging. (a) A $4 \times 1$ multiplexer with four channels (in red) and two bits $A_{1} A_{0}$ (in green) for selection. (b) The truthtable of the multiplexer in (a).

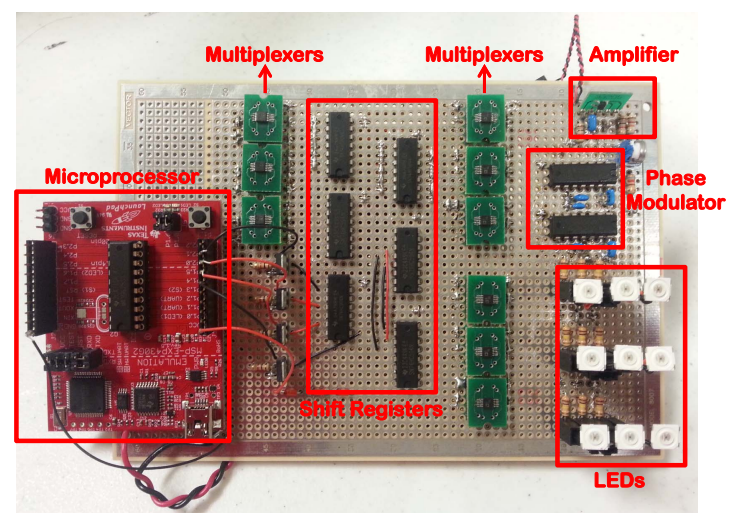

Figure 8. Phase messaging array (PMA) circuit for experiments.

\section{Experiments}

In our experiments, the receiver is a CW ToF camera SoftKinect DS311. Light from LEDs of this camera is IR with wavelength of $850 \mathrm{~nm}$ and modulation frequency $f_{m}=15 \mathrm{MHz}$. The depth sensor has a resolution of $160 \times 120$. The PMA transmitter is illustrated in Figure 8. There are five basic components: (1) photo detector and signal amplifier; (2) phase shifter; (3) multiplexers; (4) an array of 9 LEDs; (5) shift register to convert the serial control flow into the parallel one for all multiplexers. The control flow is converted into a binary stream and buffered at a microprocessor MSP430G in this experiment. For the PMA transmitter circuit, it is important to select component chips with delay far less than one period, i.e. 66ns.

We test the system in an indoor environment by sending random bits from PMA and measure the accuracy (or recognition rate) at the receiver side (i.e. ToF camera). Here, recognition rate is given by the number of correctly recognized bits divided by the number of transmitted bits. One snapshot of the captured phase image is illustrated in Figure 9(a). For message extraction, we get average phase values for each LED as in Figure 9(b) and assign phase labels by the threshold method. Refer to the truthtable in Figure 7(b) and labels $p_{2} p_{1} p_{0} p_{3} p_{2} p_{1} p_{0} p_{3} p_{1}$ are decoded to the

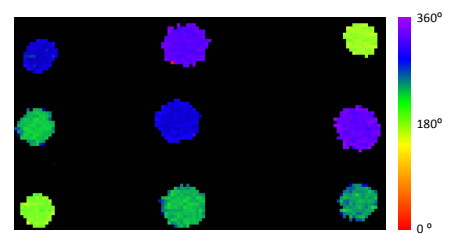

(a) Snapshot of 9 LEDs

$\begin{array}{llllll}272.6 & 323.5 & 172.4 & p_{2} & p_{3} & p_{0} \\ 232.9 & 276.8 & 327.1 & p_{1} & p_{2} & p_{3} \\ 182.3 & 236.7 & 242.3 & p_{0} & p_{1} & p_{1}\end{array}$

(b) Phase values in degree

(c) Label assignment

Figure 9. Phase symbol assignment for one phase image. (a) A phase image with 9 LEDs. For visualization, phase values are mapped to colors. (b) Mean phase values of 9 LEDs in degree. (c) Phase symbol assignment with $p_{0}-p_{3}$ by the threshold method.

binary form "100100111001001101".

Of the nine LEDs in the prototype, one LED is used as an indicator to signal the frame transition. The other eight LEDs provide 16 message bits per captured frame. We send 8000 random bits using the PMA and extract messages at the ToF camera. In the current implementation the PMA is held closely to the ToF camera for sufficiently high signal to noise ratio. The average accuracy of five independent experiments is $\mathbf{9 7 . 8} \%$ with a standard deviation of $1.3 \%$. The camera frame rate for experiments is 24 fps (framesper-second). In practice, the transmission rate at the PMA is modified to allow for repetitive frames to assist the synchronization and the measured number of repetitive frames has a mean of 6.3, resulting in an effective frame rate of 4 fps. With 16 bits per frame ( 2 bits per LED), the effective message rate is approximately 64 bps. By addressing synchronization without using repetitive frames, the data rate for this method can reach 960 bps by setting 60fps, i.e. the maximum frame rate of SoftKinect DS311. An accuracy of $97.8 \%$ corresponds to an uncoded bit error rate of $2.2 \%$. Appropriate error correction codes such as Reed-Solomon for burst error and convolutional codes for random errors can be used to improve performance of accuracy, at the tradeoff of bit rate.

\subsection{Robustness}

We compare the influence of ambient background illumination for 1) phase-based messaging with a ToF camera (SoftKinetic DS311) as the receiver and 2) intensitybased messaging using typical cameras (smart phone Samsung SIII and consumer digital camera Sony DSC-RX100) as receivers. The transmitter for phase-messaging is our PMA displaying four phases sequentially; while the transmitter for the intensity-based messaging is a visible light 
LED switching between on and off and controlled by a simple electronic timer. Plots of captured intensity and phases are shown in Figure 10. For the normal indoor condition, both ToF camera and intensity-based cameras have switching states as expected. We then apply a bright ambient illumination (from an ordinary fluorescent office light) that is manually switched on and off. With this strong timevarying background illumination, the captured values from intensity-based camera is significantly degraded. We repeat sending 8000 random bits under varying background illumination for five times. The average bit accuracy is $\mathbf{9 5 . 8} \%$ with a standard deviation of $2.1 \%$, for the phase-based messaging. The intensity-based messaging gives an accuracy of $100 \%$ with no background illumination, but the accuracy dips to approximately $70 \%$ with random blinking background illumination. As expected, the phase-based messaging method is much more robust against ambient or background illumination than intensity-based messaging.

\subsection{Data Rate Discussion}

The system we present in this paper is a complete implementation of the proof of concept $3 \times 3$ phase-messagingarray with nine transmitter LEDs. In the current implementation, each LED can emit a time varying signal taking on values from a set of four phase delays, i.e. a 2 bit binary message stream. The basic concept can be easily extended to increase the number of LEDs packed in the spatial plane and to increase the number of phase symbols. Of the nine LEDs in the prototype, eight messaging LEDs provide 16 message bits per captured frame. The ToF camera works at the framerate of $24 \mathrm{fps}$ so that the prototype can transmit up to 384 bps with synchronization, and $960 \mathrm{bps}$ at $60 \mathrm{fps}$. An extension of the current approach can be made by increasing the number of phase symbols to span the 360-degree range. With the current level of phase noise $\left(\sigma_{p}=1.2\right)$ approximately $(360 / 2.4)=150$ symbols or approximately 7 bits can be implemented and still be separated enough for discrimination. By also increasing the spatial density of the LEDs to a $100 \times 100$ array, and operating at $60 \mathrm{fps}$, the extended data rate would be 4.2 Mbps. Further modification of the hardware of the PMA to reduce the phase variance so that 256 symbols can be used would enable full utilization of the 8-bit depth camera range and give a data rate of $4.8 \mathrm{Mbps}$. A key interesting point is that this communications channel is achievable while the depth camera continues its normal operation as a depth camera. No additional hardware on the ToF camera is necessary for its role as the message receiver. As future work, phase-based messaging can be combined with varying the amplitude of the light signal to achieve a combination of both intensity-based and phase-based messaging (in environments with low ambient light). For example, with 8-bits of intensity variation, a $100 \times 100$ pixel array, and a 60 frames-per-second camera, the expected data rate would be $600000 \times 2 \times 8=9,600,000 \approx 10 \mathrm{Mbps}$.

\section{Conclusion}

In this work, we propose and develop a communication methodology with a low-cost continuous-wave ToF camera and low-cost custom circuitry. The novel method, called Phase Messaging Array (PMA), is the first of its kind that allows messaging to the ToF camera using electronically controllable phase. The message bits can be encoded temporally via phase delays and spatially in the $\mathrm{x}-\mathrm{y}$ coordinate frame. We demonstrate high accuracy messaging with the PMA operating independently from the ToF camera, suggesting applications where PMA's in scenes can act as messaging tags, beacons or landmarks for auxilliary communication to mobile systems employing depth cameras. A key demonstrated advantage of phase-messaging is the performance under various lighting conditions allowing operation in outdoor and bright light environments. Messaging accuracies for all cases are above $95 \%$.

\section{Acknowledgments}

This work is supported by the US National Science Foundation (NSF) under the grant CNS-1065463.

\section{References}

[1] ISO/IEC 18004: 2006 Information technology Automatic identification and data capture techniques QR code 2005 bar code symbology specification, 2006.

[2] J. Armstrong. Ofdm for optical communications. J. Lightwave Technol., 27(3):189-204, February 2009.

[3] A. Ashok, M. Gruteser, N. Mandayam, T. Kwon, W. Yuan, M. Varga, and K. Dana. Rate adaptation in visual mimo. Proceedings of IEEE Conference on Sensor, Mesh and Ad Hoc Communications and Networks, pages 583-591, 2011.

[4] A. Ashok, M. Gruteser, N. Mandayam, J. Silva, M. Varga, and K. Dana. Challenge: mobile optical networks through visual mimo. International conference on Mobile computing and networking, pages 105-112, 2010.

[5] A. Bhandari, A. Kadambi, R. Whyte, L. Streeter, C. Barsi, A. Dorrington, and R. Raskar. Multifrequency time of flight in the context of transient renderings. In ACM SIGGRAPH, pages 46:1-46:1, 2013.

[6] Y. Cui, S. Schuon, D. Chan, S. Thrun, and C. Theobalt. 3D shape scanning with a time-of-flight camera. In IEEE CVPR, pages 1173-1180, 2010.

[7] M. V. den Bergh and L. V. Gool. Combining RGB and ToF cameras for real-time 3D hand gesture interaction. In IEEE WACV, pages 66-72, 2011.

[8] V. Ganapathi, C. Plagemann, D. Koller, and S. Thrun. Real time motion capture using a single time-of-flight camera. In IEEE CVPR, pages 755-762, 2010.

[9] H. Haas. High-speed wireless networking using visible light. SPIE Optoelectronics and Communications, April 2013.

[10] M. Hansard, S. Lee, O. Choi, and etc. Time of Flight Cameras: Principles, Methods, and Applications. Springer, 2013.

[11] R. I. Hartley and A. Zisserman. Multiple View Geometry in Computer Vision. Cambridge University, 2004. 


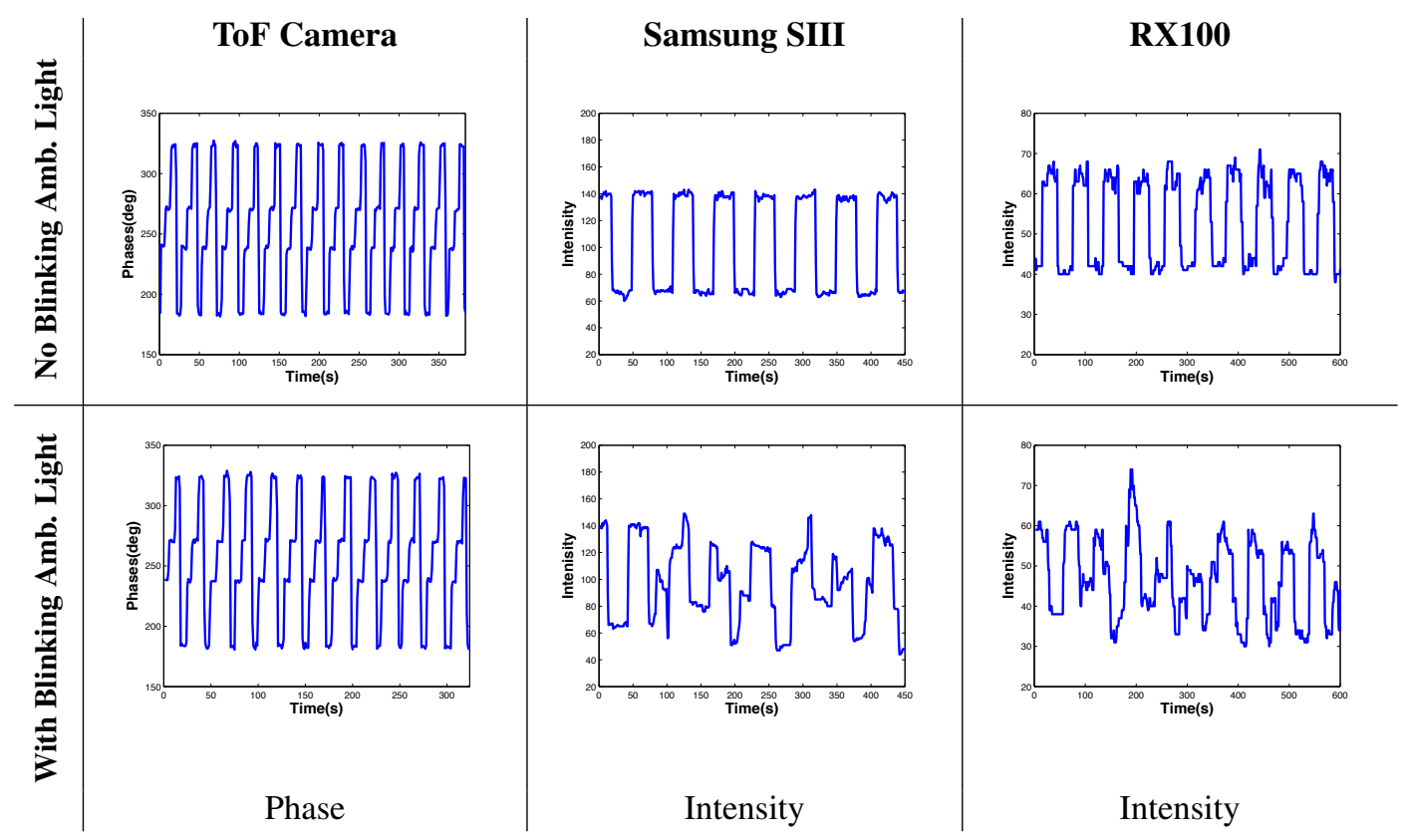

Figure 10. Influence of ambient background illumination. We compare the influence of time-varying ambient light for phase-based messaging with a ToF camera (SoftKinetic DS311) and intensity-based messaging (using a smart phone camera Samsung SIII and a consumer digital camera Sony DSC-RX100). Phase-based messaging is more robust against time-varying ambient illumination.

[12] R. P. Horaud. Tutorial on 3D Sensors, April 2013.

[13] A. Kadambi, R. Whyte, A. Bhandari, L. Streeter, C. Barsi, A. Dorrington, and R. Raskar. Coded time of flight cameras: Sparse deconvolution to address multipath interference and recover time profiles. ACM Trans. Graph., 32(6):167:1167:10, November 2013.

[14] K. Kamijo, N. Kamijo, and G. Zhang. Invisible barcode with optimized error correction. In IEEE International Conference on Image Processing, pages 2036 -2039, October 2008.

[15] X. Li, J. Vucic, V. Jungnickel, and J. Armstrong. On the capacity of intensity-modulated direct-detection systems and the information rate of aco-ofdm for indoor optical wireless applications. IEEE Transactions on Communications, 60(3):pp. 799-809, 2012.

[16] A. Mohan, G. Woo, S. Hiura, Q. Smithwick, and R. Raskar. Bokode: Imperceptible visual tags for camera based interaction from a distance. ACM Trans. Graph., 28(3), July 2009.

[17] M. R. H. Mondal and J. Armstrong. Impact of linear misalignment on a spatial OFDM based pixelated system. In Asia-Pacific Conference on Communications, 2012.

[18] A. Q. Morton. Packaging history: The emergence of the Uniform Product Code (UPC) in the United States. History and Technology, 11(1):101111, 1994.

[19] C. D. Mutto, P. Zanuttigh, and G. M. Cortelazzo. Time-ofFlight Cameras and Microsoft Kinect(TM). Springer Publishing Company, Incorporated, 2012.

[20] N. Naik, S. Zhao, A. Velten, R. Raskar, and K. Bala. Single view reflectance capture using multiplexed scattering and time-of-flight imaging. In Proceedings of SIGGRAPH Asia Conference, pages 171:1-171:10, 2011.
[21] S. D. Perli, N. Ahmed, and D. Katabi. Pixnet:designing interference-free wireless links using lcd-camera pairs. ACM Int. Conf. on Mobile Computing and Networking, 2010.

[22] S. Schmid, G. Corbellini, S. Mangold, and T. R. Gross. Ledto-led visible light communication networks. In Proceedings of the fourteenth ACM international symposium on Mobile ad hoc networking and computing (MobiHoc), 2013.

[23] A. Velten, T. Willwacher, O. Gupta, A.Veeraraghavan, M.G.Bawendi, and R. Raskar. Recovering three-dimensional shape around a corner using ultrafast time-of-flight imaging. Nature Communication, 3:745+, 2012.

[24] J. Wakerly. Digital Design: Principles and Practices (4th Edition). Prentice-Hall, Inc., 2005.

[25] T. Q. Wang, Y. A. Sekercioglu, and J. Armstrong. Analysis of an optical wireless receiver using a hemispherical lens with application in mimo visible light communications. $J$. Lightwave Technol., 31(11):1744-1754, June 2013.

[26] D. Wu, M. O'Toole, A. Velten, A. Agrawal, and R. Raskar. Decomposing global light transport using time of flight imaging. In IEEE CVPR, pages 366-373, 2012.

[27] W. Yuan, K. Dana, A. Ashok, M. Varga, M. Gruteser, and N. Mandayam. Photographic steganography for visual mimo: A computer vision approach. IEEE WACV, 2012.

[28] W. Yuan, K. Dana, A. Ashok, M. Varga, M. Gruteser, and N. Mandayam. Spatially varying radiometric calibration for camera-display messaging. IEEE GlobalSIP, 2013.

[29] W. Yuan, K. Dana, M. Varga, A. Ashok, M. Gruteser, and N. Mandayam. Computer vision methods for visual mimo optical system. In IEEE Computer Society Conference on Computer Vision and Pattern Recognition Workshops (CVPRW), pages 37-43, 2011. 